\title{
PLANE CONTACT OF TWO ELASTIC SOLIDS WITH FUNCTIONALLY GRADED COATINGS JOINED BY AN IMPERFECT INTERFACE
}

\author{
S. M. Aizikovich ${ }^{*}$, S. S. Volkov**, A. S. Vasiliev**
}

\begin{abstract}
Plane contact problem on normal interaction of two dissimilar elastic solids is considered. The solids consist of a homogeneous semi-infinite substrate and a functionally graded coating joined by an imperfect interface to the substrate. The problem is reduced to the solution of a dual integral equation which is solved by the bilateral asymptotic method. Numerical results illustrating the difference between the ideal and imperfect interface of the coating and the substrate are provided.
\end{abstract}

Keywords: contact, elasticity, functionally graded coating, imperfect interface, analytical method

\section{Introduction}

Most of the results in the field of contact mechanics of elastic solids with functionally graded (FG) and homogeneous coatings are obtained in assumption of complete adhesion (perfect coupling) at the interface between the coating and substrate (Guler and Erdogan, 2004, Ke and Wang, 2006). However, the coating-substrate adhesion depends on the technique of coating application and on the existence of microdefects on this interface. Some results in contact interaction of solids with homogeneous and piecewise homogeneous coatings taking into account imperfect coating-substrate interface one can find in the papers by Antonenko and Velichko (2014); Lipton (2001); Torskaya and Goryacheva (2003) and We et al. (2013), etc. The present paper addresses to the study of contact interaction of coated FG solids.

\section{Statement of the problem}

Let us consider plane contact of two massive elastic parabolic solids with coatings, initially contacting at a point $(0,0)$ of a Cartesian coordinate system $(x, z)$. The solids are subjected to the normal centrally applied force $P$ and move distances $-\delta_{1}$ and $\delta_{2}$ along the $z$-axis. Hereafter indexes 1 and 2 correspond to the lower and upper solid, respectively. Displacements of the solid's surface can be expressed by the following equation:

$$
z=0: w_{1}-x^{2}\left(2 R_{1}\right)^{-1}+\delta_{1}=w_{2}+x^{2}\left(2 R_{2}\right)^{-1}-\delta_{2}
$$

where $R_{1}$ and $R_{2}$ are radii of curvature of the solids, $u$ and $w$ are displacements with respect to $x$ and $z$.

Each solid consist of a homogeneous half-plane (substrate) with constant values of elastic moduli $E_{i}^{(s)}, v_{i}^{(s)}$ and a functionally graded coating of thickness $H_{i}$. Young's modulus and Poisson's ratio of the coating vary with depth according to the continuously differentiable functions $E_{i}^{(c)}(z), v_{i}^{(c)}(z)$. Hereafter,

Prof. Sergei Aizikovich, DSc.: Research and Education Center "Materials", Don State Technical University, 1 Gagarin sq., Rostov-on-Don, Russia, saizikovich@gmail.com

** Senior Research Fellows Andrey Vasiliev and Sergei Volkov, PhD.: Research and Education Center "Materials", Don State Technical University, 1 Gagarin sq., Rostov-on-Don, Russia; andre.vasiliev@gmail.com 
superscripts $(c)$ and $(s)$ correspond to the coating and to the substrate, respectively. Let us consider imperfect coupling of the coating and substrate:

$$
z=(-1)^{i} H_{i}: \quad \sigma_{z}^{(c)}=\sigma_{z}^{(s)}, w^{(c)}=w^{(s)}, \tau_{z x}^{(c)}=\tau_{z x}^{(s)}, \tau_{z x}^{(c)}=\frac{u^{(c)}-u^{(s)}}{e_{i}}, \quad i=1,2
$$

where $\sigma_{z}$ and $\tau_{\mathrm{zx}}$ are the components of the stress tensor, coefficients $e_{i} \in[0, \infty]$ characterizes the adhesion at the interface. The case of $e_{i}=0$ correspond to the comlete perfect coupling, i.e. $u^{(c)}=u^{(s)}$. The case of $e_{i}=\infty$ correspond to the full slip. Methods for determining this coefficient are discussed in (Goryacheva, 1998).

Outside of the punch, the surface is traction-free. It is required to determine the contact normal stresses under the punch: $\left.\sigma_{z}^{(c)}\right|_{z=0}=p(x), x \leq a$ and the radius of the contact area $a$.

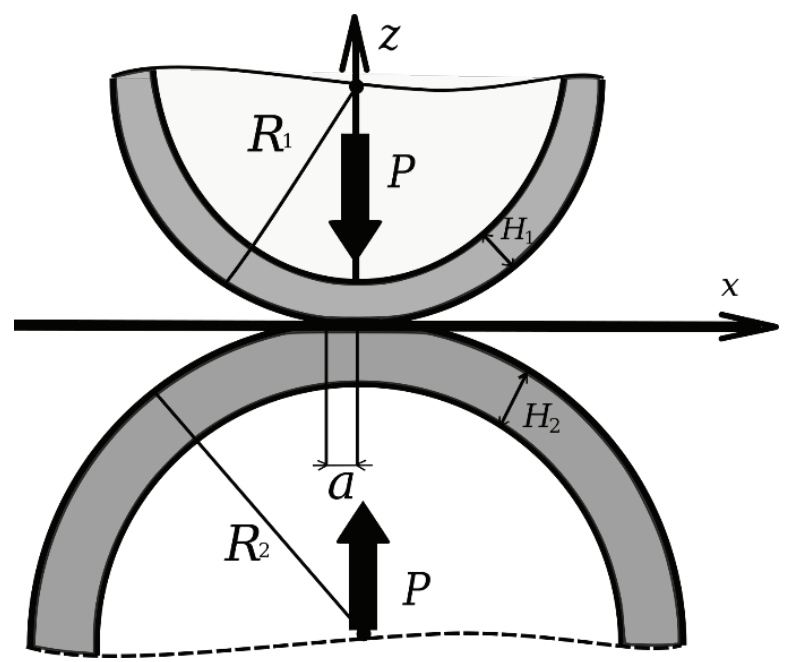

Fig. 1: Scheme of the contact problem

\section{Solution of the problem}

Using the Fourier integral transformation technique, similar to the axisymmetric formulation (Kudish et al., 2017), the dual integral equation of the problem is obtained:

$$
\begin{gathered}
\int_{0}^{\infty} L(\lambda \gamma) \frac{\bar{p}_{0}(\gamma)}{\gamma} \cos \left(\gamma x^{\prime}\right) d \gamma=\frac{\pi \Theta_{c}}{2 a}\left(\delta_{1}+\delta_{2}-\frac{a^{2} x^{\prime 2}}{2 R}\right),\left|x^{\prime}\right| \leq 1 \\
L(\lambda \gamma)=\Theta_{c}\left(\frac{L_{1}(\lambda \gamma)}{E_{1}^{\prime(c)}}+\frac{L_{2}(\lambda \mu \gamma)}{E_{2}^{\prime(c)}}\right), \quad \Theta_{c}=\left(\frac{1}{E_{1}^{\prime(c)}}+\frac{1}{E_{2}^{\prime(c)}}\right)^{-1}, R=\left(\frac{1}{R_{1}}+\frac{1}{R_{2}}\right)^{-1} \\
E_{i}^{\prime(c)}=E_{i}^{(c)}(0) /\left(1-v_{i}^{(c)}(0)\right), \quad E_{i}^{(s)}=E_{i}^{(s)} /\left(1-v_{i}^{(s)}\right)
\end{gathered}
$$

where $x^{\prime}=x / a$ is the dimensionless coordinate; $\lambda=H_{1} / a$ is the relative thickness of the lower coating; $\mu=H_{2} / H_{1}$ is the ratio of the coating's thicknesses; $E_{i}^{\prime(c)}$ are the effective elastic moduli on the surface of the coatings; $\bar{p}_{0}(\gamma)$ is the Fourier transform of the dimensionless contact stresses $p_{0}\left(x^{\prime}\right)=p\left(a x^{\prime}\right) ; L_{i}(\lambda \gamma)$ are the compliance functions of the lower and upper solids. Compliance functions are defined as solutions of two-point boundary value problems for a system of ordinary differential equation with variable coefficients, which can be obtained similar to (Vasiliev et al., 2017a). 
To illustrate how the imperfect coupling of the coating and substrate influence the fundamental characteristics of the contact let us consider following example:

$$
\begin{gathered}
v_{i}^{(c)}(z) \equiv v_{i}^{(s)}=0.3, \quad E_{1}^{(s)}=E_{2}^{(s)}=E_{0}, \quad \mu=H_{2} / H_{1}=0.25, \quad e_{1}=e_{2} \\
E_{1}^{(c)}(z)=0.5 E_{0}\left(1-z / H_{1}\right),-H_{1} \leq z \leq 0 ; \quad E_{2}^{(c)}(z)=E_{0}\left(2-z / H_{2}\right), 0 \leq z \leq H_{2}
\end{gathered}
$$

Figure 2 contain the graphs of the kernel transform $L(\lambda \gamma)$ for different values of parameters $e_{\mathrm{i}}$. It is seen that the adhesion coefficient sufficiently change the behavior of the kernel transform. As it was shown earlier behavior of the kernel transform significantly affects the behavior of the most important characteristics of the contact such as contact stresses, size of the contact area, contact stiffness, etc., see (Vasiliev et al., 2017b) for details.

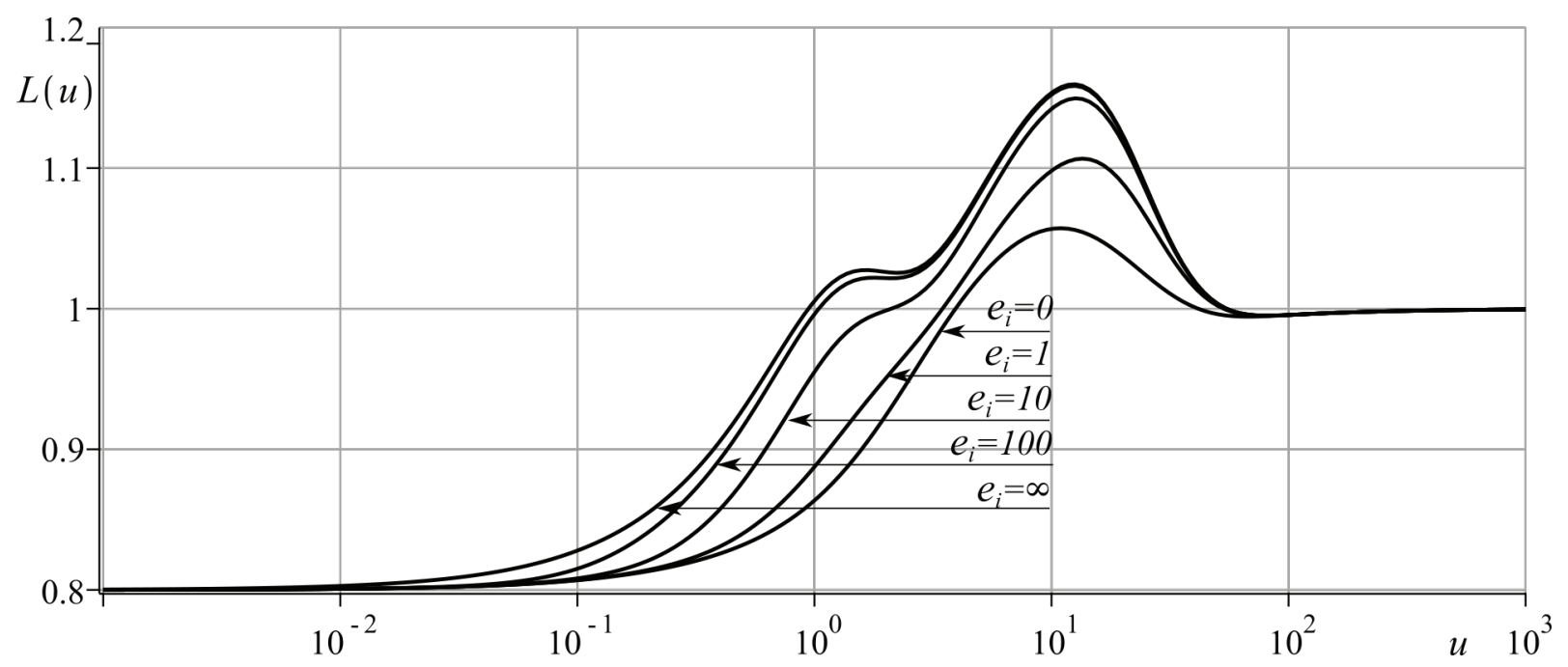

Fig. 1: Kernel transform for different values of adhesion coefficient $e_{i}=0,1,10,100$ and $\infty$.

Solution of the dual integral equation (3) is constructed using the bilateral asymptotic method. Kernel transform $L(\lambda \gamma)$ is approximated by the following expression for a fixed value of $\mu$ :

$$
L(\lambda \gamma) \approx \Pi_{N}(\lambda \gamma)=\prod_{i=1}^{N}\left(\lambda^{2} \gamma^{2}+A_{i}^{2}\right) /\left(\lambda^{2} \gamma^{2}+B_{i}^{2}\right), \quad A_{i}, B_{i} \in C .
$$

Earlier it was shown for the vast majority of types of depth-wise variation of elastic moduli that an approximation with relative error less that $0.5 \%$ can be constructed.

Taking into account classical assumption for the contact stresses at the boundary of the contact area: $p_{0}(1)=0$, similar to (Vasiliev et al., 2017) approximated analytical expressions for the contact stresses are obtained:

$$
\begin{gathered}
p_{0}\left(x^{\prime}\right)=\frac{2 P}{a \pi}\left[\sqrt{1-x^{\prime 2}}+\sum_{i=1}^{N} C_{i}\left(2 \frac{\lambda}{A_{i}} \sqrt{1-x^{\prime 2}}-Z\left(\frac{A_{i}}{\lambda}, x^{\prime}\right)\right)\right] \\
Z(a, x)=\int_{x}^{1} \frac{t \cosh (a(t-x)) I_{0}(a) / I_{1}(a)-\sinh (a(t-x))}{\sqrt{1-t^{2}}} d t
\end{gathered}
$$

Parameters $C_{i}$ are the solution of the following system of linear algebraic equations:

$$
\sum_{i=1}^{N} C_{i}\left(\frac{B_{k} I_{0}\left(A_{i} \lambda^{-1}\right) / I_{1}\left(A_{i} \lambda^{-1}\right)+A_{i} K_{0}\left(A_{i} \lambda^{-1}\right) / K_{1}\left(A_{i} \lambda^{-1}\right)}{A_{i}^{2}-B_{k}^{2}}+2 \lambda\right)=-\frac{1}{B_{k}}, k=1 . . N
$$

Half width of the contact area satisfy following equation: 


$$
a^{2}=\frac{4 P R}{\pi \Theta_{s}}\left(1+2 \lambda \sum_{i=1}^{N} \frac{C_{i}}{A_{i}}\right)
$$

Expressions (8) and (11) are asymptotically exact for small and large values of dimensionless coating thickness $\lambda$.

\section{Conclusions}

Two-dimensional (plane) contact problem on interaction of two dissimilar elastic solids with FG coatings is considered under the assumption, that coating-substrate interface is imperfect. Approximated analytical expressions for the contact stresses and size of the contact area are obtained. Using these results internal stresses within the coating and substrate can be easily calculated, see (Volkov et al., 2016) for details. The results can be generalized to the case of elastohydrodynamic lubricated contact (Kudish et al., 2016), electroelastic piezoelectric materials (Volkov et al., 2017) or to the case of bending of a flexible element (beam, plate) lying of a FG coating-substrate system (Volkov and Vasiliev, 2014).

\section{Acknowledgement}

Authors acknowledge the support of the Russian Foundation for Basic Research (RFBR) (grant numbers 16-07-00958-a, 18-07-01177-a) and the scholarship of the President of the Russian Federation (SP3615.2018.1).

\section{References}

Antonenko, N.N. and Velichko, I.G. (2014) Contact Problem of Torsion of a Multilayer Base with Elastic Connections between Layers. Vestn. Samar. Gos. Tekhn. Univ. 36, pp. 66-78, doi: 10.14498/vsgtu1319

Goryacheva, I.G. (1998) Contact Mechanics in Tribology, Kluwer Academic Publishers, Dordrecht.

Guler M.A., Erdogan F. Contact mechanics of graded coatings // Int. J. Solids Struct. 2004. Vol. 41, pp. 2865-3889.

Ke L.-L., Wang Y.-S. Two-dimensional contact mechanics of functionally graded materials with arbitrary spatial variations of material properties // Int. J. Solids Struct. 43 (2006) 5779-5798.

Kudish, I.I., Volkov, S.S., Vasiliev, A.S. and Aizikovich, S.M. (2016). Some criteria for coating effectiveness in heavily loaded line EHL contacts. Part 2. Lubricated contacts. ASME Journal of Tribology, 138, doi: 10.1115/1.4030958.

Kudish, I.I., Volkov, S.S., Vasiliev, A.S. and Aizikovich, S.M. (2017) Lubricated Point Heavily Loaded Contacts of Functionally Graded Materials. Part 1. Dry Contacts. Mathematics and Mechanics of Solids, doi: $10.1177 / 1081286517704689$

Lipton, R. (2001) Reinforcement of elastic structures in the presence of imperfect interfacial bonding Journal of Engineering Mechanics, 127(7), pp. 667-671

Torskaya, E.V. and Goryacheva, I.G. (2003) The effect of interface imperfection and external loading on the axisymmetric contact with a coated solid. Wear, 254, pp. 538-545

Vasiliev, A.S., Volkov, S.S., Aizikovich, S.M. and Mitrin, B.I. (2017a) Plane contact problem on indentation of a flat punch into a transversely-isotropic half-plane with functionally graded transversely-isotropic coating. Zeitschrift für angewandte Mathematik und Physik., 68, doi: 10.1007/s00033-016-0746-8.

Vasiliev, A.S., Volkov, S.S., Belov, A.A., Litvinchuk, S.Yu. and Aizikovich, S.M. (2017b) Indentation of a hard transversely isotropic functionally graded coating by a conical indenter. International Journal of Engineering Science, 112, pp. 63-75.

Volkov, S.S. and Vasiliev, A.S. (2014). Mathematical modeling of interaction of a circular plate with an elastic inhomogeneous layer. Springer Proceedings in Physics 152, pp. 223-229.

Volkov, S.S., Vasiliev, A.S., Aizikovich, S.M., Seleznev, N.M. and Leontieva, A.V. (2016) Stress-strain state of an elastic soft functionally-graded coating subjected to indentation by a spherical punch. PNRPU Mechanics Bulletin 4, pp. 20-34.

Volkov, S.S., Vasiliev, A.S., Aizikovich, S.M. and Mitrin, B.I. (2017) Axisymmetric indentation of an electroelastic piezoelectric half-space with functionally graded piezoelectric coating by a circular punch. Acta Mechanica, pp. 1-17, doi: 10.1007/s00707-017-2026-x

Wu, Y.F., Yu, H.Y. and Chen, W.Q. (2013) Indentation responses of piezoelectric layered half-space. Smart Materials and Structures. 2013. T. 22. C. 1-16. doi:10.1088/0964-1726/22/1/015007 